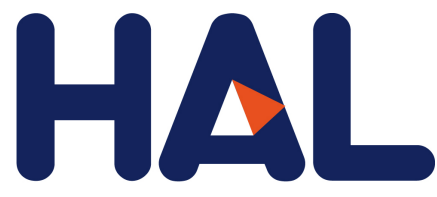

archives-ouvertes.

\title{
Grayness-dependent emission of dispersive waves from dark solitons in optical fibers
}

T. Marest, C. Mas Arabí, M. Conforti, A. Mussot, C. Milián, D. Skryabin, A. Kudlinski

\section{- To cite this version:}

T. Marest, C. Mas Arabí, M. Conforti, A. Mussot, C. Milián, et al.. Grayness-dependent emission of dispersive waves from dark solitons in optical fibers. Optics Letters, Optical Society of America, 2018, 43 (7), pp.1511. 10.1364/OL.43.001511 . hal-02384998

\section{HAL Id: hal-02384998 \\ https://hal.archives-ouvertes.fr/hal-02384998}

Submitted on 28 Nov 2019

HAL is a multi-disciplinary open access archive for the deposit and dissemination of scientific research documents, whether they are published or not. The documents may come from teaching and research institutions in France or abroad, or from public or private research centers.
L'archive ouverte pluridisciplinaire HAL, est destinée au dépôt et à la diffusion de documents scientifiques de niveau recherche, publiés ou non, émanant des établissements d'enseignement et de recherche français ou étrangers, des laboratoires publics ou privés. 


\title{
Grayness-dependent emission of dispersive waves from dark solitons in optical fibers
}

\author{
T. Marest ${ }^{1}$, C. Mas Arabí ${ }^{1}$, M. Conforti $^{1}$, A. Mussot ${ }^{1}$, C. Milián ${ }^{2}$, D.V. Skryabin ${ }^{2,3}$, And A. \\ KUDLINSKI ${ }^{1, *}$ \\ ${ }^{1}$ Univ. Lille, CNRS, UMR 8523 - PhLAM - Physique des Lasers Atomes et Molécules, F-59000 Lille, France \\ ${ }^{2}$ Department of Physics, University of Bath, Bath BA2 7AY, United Kingdom \\ ${ }^{3}$ ITMO University, St. Petersburg 197101, Russian Federation \\ *Corresponding author: alexandre.kudlinski@univ-lille1.fr
}

Compiled February 26, 2018

\begin{abstract}
We report the experimental observation of dispersive wave emission from gray solitons propagating in the normal dispersion region of an optical fiber. Besides observing for the first $t$ ime the e mission of a dispersive wave from an isolated dark soliton, we show that the dispersive wave frequency and amplitude strongly depends on soliton grayness. This process can be explained by the higher-order dispersion contribution into the phase-matching condition and the grayness of the soliton. Numerical simulations and theoretical predictions are in good agreement with the experiments.
\end{abstract}

OCIS codes: (190.4370) Nonlinear optics, fibers; (190.5530) Pulse propagation and temporal solitons.

http://dx.doi.org/10.1364/ao.XX.XXXXXX

Temporal solitons in optical fibers are stationary solutions of the integrable nonlinear Schrödinger equation (NLSE) resulting from a perfect balance between group velocity dispersion and Kerr nonlinearity induced self-phase modulation [1, 2]. One can differentiate between two main soliton families: bright and dark solitons [3]. The bright ones are intense pulses of light propagating in anomalous dispersion region. The dark ones exhibit an intensity dip (associated with a phase jump) over a uniform background. Dark solitons having a zero intensity at their core and an abrupt $\pi$ phase jump are called black solitons or fundamental dark solitons. When the minimum intensity does not drop to zero and the phase change is smooth and smaller than $\pi$, they are called gray solitons. Both black and gray solitons are solutions of the integrable NLSE only in normal dispersion (focusing) regime [2, 3].

In optical fibers however, there are many situations in which the integrability of NLSE is broken by perturbations. For example, when the pump wavelength lies in the vicinity of the zero dispersion wavelength (ZDW), intrinsic third-order dispersion (TOD) is not negligible and has to be taken into account [4-6]. This results in the emission of radiation from both bright and dark types of solitons. In the case of bright solitons, the emission of resonant radiations (also referred to as dispersive waves or Cherenkov radiations) due to TOD has been extensively studied both from theoretical and experimental points of view [6-8]. In the case of dark solitons, the emission of dispersive radiation due to TOD has been studied theoretically and numerically in Refs. [9-11] but experiments were limited to a specific case in which a train of multiple gray solitons (with uncontrolled grayness) emits multiple dispersive waves [12]. The emission of dispersive wave from a dark soliton with well controlled grayness has never been reported experimentally. The lack of such experiments regarding dark solitons is due to the fact that their generation is much less straightforward than for bright solitons since it requires an input field with a local change of both intensity and phase [3].

In this Letter, we propose a simple technique to generate the complex fields suitable to excite black and gray solitons in an optical fiber, using commercially available waveshapers. This allows to efficiently control the soliton grayness and study its impact on the dispersive wave emission process occurring in the vicinity of the ZDW. An optimum in the efficiency of the emission process is found, in good agreement with theory and numerical simulations of the generalized NLSE.

Let us first focus on the generation of dark solitons in optical fibers. As recalled above, to create a single dark soliton, one needs to prepare an odd-symmetry dark pulse, i.e. a background field containing an intensity dip and an appropriate phase jump [13-15]. Indeed, any even-symmetry dark pulse (not containing the required phase jump, but having instead a symmetric phase with regards to the dip) will split into a pair of gray solitons [1618]. The phase profile of the input pulse is therefore of crucial importance for the generation of single black and gray solitons [3]. Several passive pulse shaping methods based on frequency and phase filtering using spatial masks $[19,20]$ have been proposed to synthesize the odd dark pulses required to excite dark solitons [14, 15]. Here, we use commercially available waveshapers (Finisar WaveShaper 4000S) to synthesize a single odd dark pulse with the required amplitude and phase profiles over a background pulse [21]. More precisely, we generate pulses with the amplitude $A(t)=\sqrt{P_{0}} A_{D}(t) . A_{B G}(t)$ where $P_{0}$ is the peak power, $A_{D}(t)$ is the shape of the $\operatorname{dip}$ and $A_{B G}(t)$ is the 


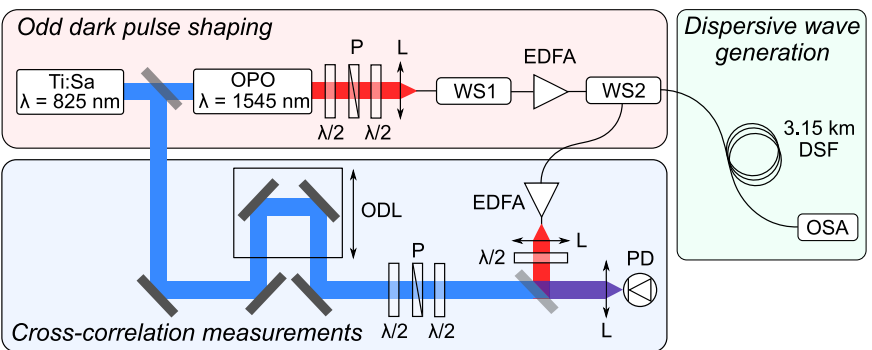

Fig. 1. Experimental setup used to characterize the shaped odd dark pulses and to investigate the generation of dispersive waves from dark solitons. ODL: optical delay line; $\lambda / 2$ : half-wave plate; P: polarizer; L: lens; WS: waveshaper; EDFA: erbium-doped fiber amplifier; OSA: optical spectrum analyzer; PD: photodiode.

background pulse, taking the form

$$
\begin{gathered}
A_{D}(t)=\cos \varphi \tanh \left(\frac{t}{T_{0}} \cos \varphi\right)-i \sin \varphi \\
A_{B G}(t)=\exp \left(-\left(\frac{t}{T_{B G}}\right)^{n}\right)
\end{gathered}
$$

where $T_{0}$ is the duration of the dark pulse, $-\pi / 2<\varphi<\pi / 2$ is the grayness parameter of the dark pulse $(\varphi=0$ corresponding to the black soliton) and $T_{B G}$ is the duration of the superGaussian background pulse (with $n=16$ ). The use of a background pulse instead of a cw one allows to avoid any possible stimulated Brillouin scattering effect.

The experimental setup is shown in Fig. 1. An optical parametric oscillator (pumped by a Ti:Sa laser) delivers Gaussian pulses tunable around $1550 \mathrm{~nm}$ with a full width at half maximum (FWHM) duration of 220 fs close to the Fourier-transform limit. The power and polarization state of the beam are controlled with a combination of half-wave plate and polarizer. The pulses then pass through a combination of waveshapers and amplifiers where they are shaped in amplitude and phase to obtain the required odd dark pulse onto a background pulse as described above. More precisely, the first waveshaper WS1 is used to shape the amplitude of the dark pulse over the superGaussian background pulse [see examples in Figs. 2(a) and (e)], without any control of the phase at this stage. Here we take $T_{0}=600 \mathrm{fs}, T_{B G}=40 T_{0}=24 \mathrm{ps}$ and $n=16$. The resulting pulse is then amplified and sent into the second waveshaper WS2, which is used to shape the required odd-symmetry phase profile [see examples in Figs. 2(b) and (f)]. Additionally to this phase shaping, WS2 is used to filter out the amplified spontaneous emission generated in the EDFA and correct amplitude distortions due to amplification. The control of amplitude and phase with two distinct waveshapers before and after amplification allows to reduce distortions of the phase profile occurring in the amplifier. Finally, the shaped pulses are launched in a dispersion shifted fiber (DSF) in which the dark solitons are expected to be excited.

The part of Ti:Sa laser beam at $825 \mathrm{~nm}$ not pumping the OPO is taken to perform cross-correlation measurements (blue beam in Fig. 1) of the shaped pulses using the two photon absorption process in a photodiode [22]. This characterization requires a power level substantially higher than required for the excitation of dark solitons in the fiber, so that a second EDFA is needed to amplify the odd dark pulse before the cross-correlation measurement in the photodiode. As discussed above, this leads to
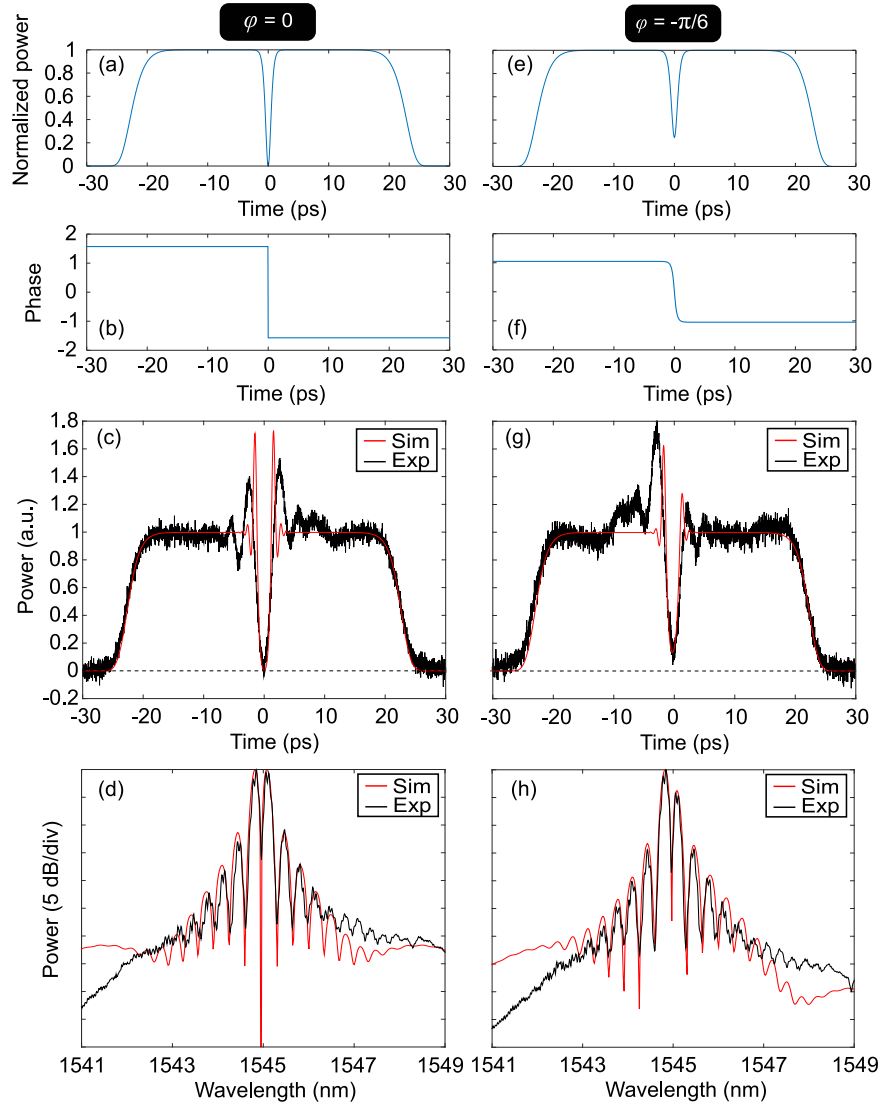

Fig. 2. (a), (b) Intensity and phase profile applied to WS1 and WS2, respectively, to generate a dark pulse with $\varphi=0$. (c) Cross-correlation trace measured after the second EDFA (black line) and simulated one (red line). (d) Measured spectrum (black line) and simulated one (red line). (e)-(h) Same plots for $\varphi=-\pi / 6$.

distortions of the phase profile, but this still allows to characterize properly the intensity. Two examples of cross-correlation measurements corresponding to $\varphi=0$ and $\varphi=-\pi / 6$ are shown in solid black lines in Figs. 2(c) and (g) respectively. For these measurement, the central wavelength of the dark pulses was $1550 \mathrm{~nm}$. The intensity dip of the pulses drops down to zero for $\varphi=0$ (black soliton), but not for $\varphi=-\pi / 6$ (gray soliton), as expected. It is important to note that these pulses are characterized at the output of a $14 \mathrm{~m}$ long section of single mode fiber (waveshapers, EDFA and patch cords linking them) with large anomalous dispersion around $1550 \mathrm{~nm}$, where dark solitons cannot be excited. This possibly explains the deformations experienced by the pulses, such as the presence of spurious peaks around the intensity dip. To confirm this, we performed numerical simulations using the generalized NLSE in the $14 \mathrm{~m}$ long single mode fiber section including the waveshapers and the EDFAs (neglecting the gain of the latter):

$$
i \partial_{z} A-\frac{\beta_{2}}{2} \partial_{t}^{2} A-i \frac{\beta_{3}}{6} \partial_{t}^{3} A+\gamma A|A|^{2}=0
$$

where $\beta_{2}=-18.3 \mathrm{ps}^{2} / \mathrm{km}$ and $\beta_{3}=0.1 \mathrm{ps}^{3} / \mathrm{km}$ are the second and third order dispersion terms and $\gamma=2 \mathrm{~W}^{-1} \cdot \mathrm{km}^{-1}$ is the nonlinear parameter of the single mode fiber. The simulated temporal profiles obtained for a peak power of $74 \mathrm{~W}$ and a central wavelength of $1550 \mathrm{~nm}$ are plotted in red lines in Figs. 

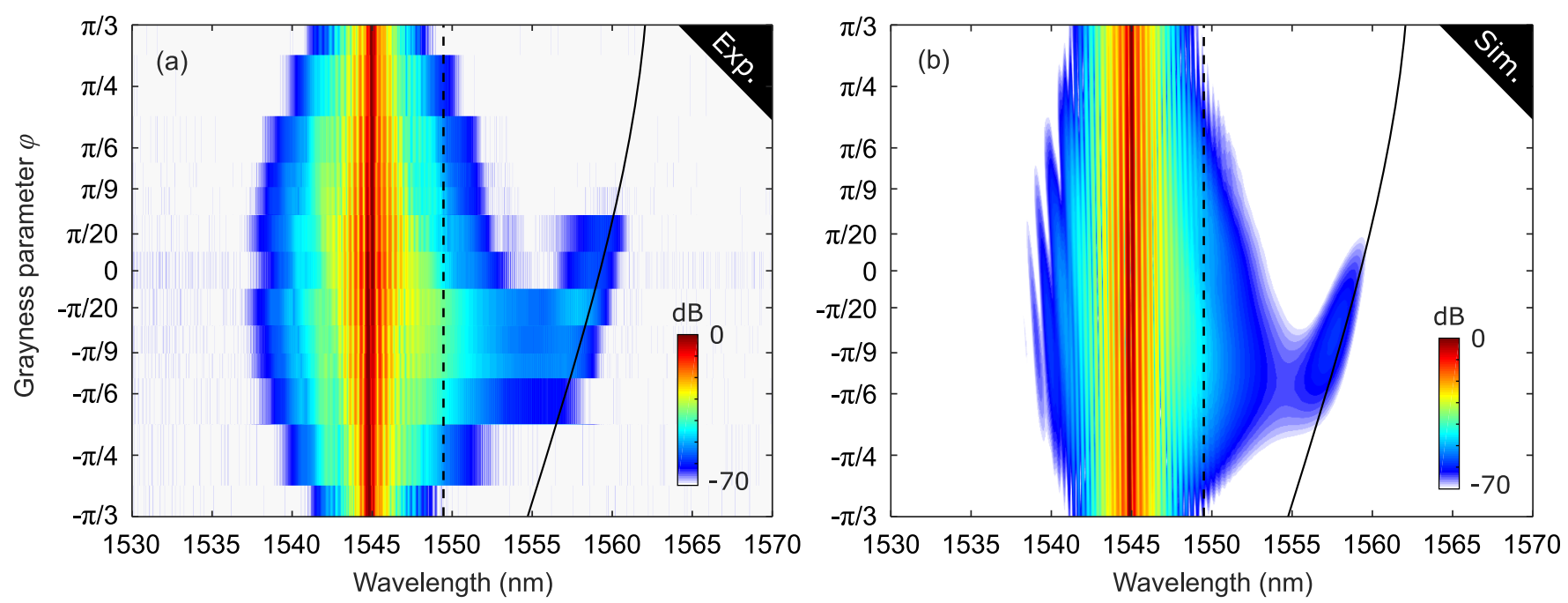

Fig. 3. (a) Measurement and (b) numerical simulation of the output spectrum for different values of the grayness parameter $\varphi$. The dashed black line represents the fiber ZDW. The solid black is the dispersive wave wavelength (solution of Eq. 4).

2(c) and (g), and show good agreement with the measured ones. In particular, they exhibit the peaks surrounding the intensity dip, with an asymmetry in the $\varphi=-\pi / 6$ case. We have checked that numerical simulations reproduce well these distortions for all other $\varphi$ values investigated hereafter. Figures 2(d) and (h) show the measured (black lines) and simulated spectra (red lines) for $\varphi=0$ and $\varphi=-\pi / 6$ respectively, in excellent agreement. As expected, the spectrum is symmetric for $\varphi=0$ but not for $\varphi=-\pi / 6$. These measurements confirm that the generated odd dark pulses have the expected properties to excite dark solitons [in the form of Eq. (1)] in the DSF, which is the next step.

The DSF is $3.15 \mathrm{~km}$ long and has a ZDW of $1549.5 \mathrm{~nm}$. The second and third-order dispersion parameters are respectively $\beta_{2}=0.417 \mathrm{ps}^{2} / \mathrm{km}$ and $\beta_{3}=0.115 \mathrm{ps}^{3} / \mathrm{km}$, and the nonlinear parameter is $\gamma=2 \mathrm{~W}^{-1} \cdot \mathrm{km}^{-1}$. In order to excite dark solitons and observe emission of dispersive waves, the pump pulses have to be launched in weak normal dispersion region. In the following experiments they were tuned to a central wavelength of $1544.9 \mathrm{~nm}$ and the second EDFA was removed since the pulses going out of WS2 are powerful enough to excite dark solitons. In this way the deformations of the ideal input pulses [Figs. 2(a-b)] are expected to desappear. The peak power $P_{0}$ was fixed to 0.58 W. Figure 3(a) shows the evolution of the output DSF spectrum as a function of the soliton grayness parameter $\varphi$ varying from $-\pi / 3$ to $+\pi / 3$. For each measurement, the driving parameters of WS1 and WS2 were finely adjusted to generate the odd dark pulse required to excite the corresponding black or gray solitons. Although we could not perform cross correlation measurements at the DSF output due to power levels being too low, the spectral measurements were consistent with the ones of dark solitons. In addition to the dark soliton spectra centered around $1544.9 \mathrm{~nm}$, a radiation is observed across the ZDW (depicted by the vertical dashed line) for grayness parameters values between $\varphi=-\pi / 6$ and $\varphi=\pi / 20$. The spectral location of this radiation changes with the grayness parameter and matches very well the black solid line representing the theoretical phase-matching relation between dark solitons and dispersive waves [9-12, 23, 24]

$$
\frac{\beta_{2}}{2} \Omega^{2}+\frac{\beta_{3}}{6} \Omega^{3}-\Omega / V_{s}+\gamma P_{0}=0
$$

where $\Omega=\omega-\omega_{0}$ is the detuning from the dark soliton central frequency $\omega_{0}$ and $V_{s}$ is the soliton velocity relatively to the pump pulse, linked to the grayness parameter through $V_{s}=1 /\left(\sqrt{\gamma \beta_{2} P_{0}} \sin \varphi\right)$. Note that Eq. (4) is an approximation following of a more general condition [11] predicting a second much weaker resonance. Equation 4 indicates that the dispersive wave frequency depends on the grayness parameter. From the good agreement between experiments and theory, we can identify the observed radiations as dispersive waves generated by dark solitons perturbed by TOD around the ZDW. In particular, these experiments show the first observation of dispersive wave emission from a black soliton. Numerical simulations corresponding to these experiments are displayed in 3(b). They were performed using Eq. 3 with the experimental and fiber parameters given above. We checked numerically that the inclusion of dispersion terms with order higher than 3 as well as self-steepening and Raman effects do not play any significant role in the dynamics. They are in excellent overall agreement with the measurements. In particular, they show that the emitted radiation closely follows the phase-matching relation, and confirm that they are emitted over a restricted range of grayness parameter value $\varphi$ and with a different efficiency from about $-\pi / 6$ to $\pi / 20$. We will now focus on this last observation.

The amplitude of the dispersive wave can be calculated by adapting the theory developed for bright solitons in [25] to the case of dark solitons. We omit here the details of the derivation, because it closely follows the bright soliton case, and report only the result. The dispersive wave in the temporal domain consists of a non-zero oscillating tail that propagates over a continuous background whose extension spans from the dark soliton to the time delay of a linear wave with the frequency of radiation [10]. The amplitude of this tail $|G|$ is given by

$$
|G|=\left|\frac{\sqrt{P_{0}} \pi \cos ^{3}(\varphi) \beta_{3}}{6 T_{0}^{2} \tilde{D}^{\prime}(\Omega)} \Delta \tilde{\omega}^{3} \sinh ^{-1}\left(\frac{\pi \Delta \tilde{\omega}}{2}\right)\right|
$$

where

$$
\Delta \tilde{\omega}=\left(\frac{\Omega T_{0}}{\cos (\varphi)}+\frac{2 \gamma P_{0} T_{0} \cos (\varphi)}{\tilde{D}^{\prime}(\Omega)}\right)
$$




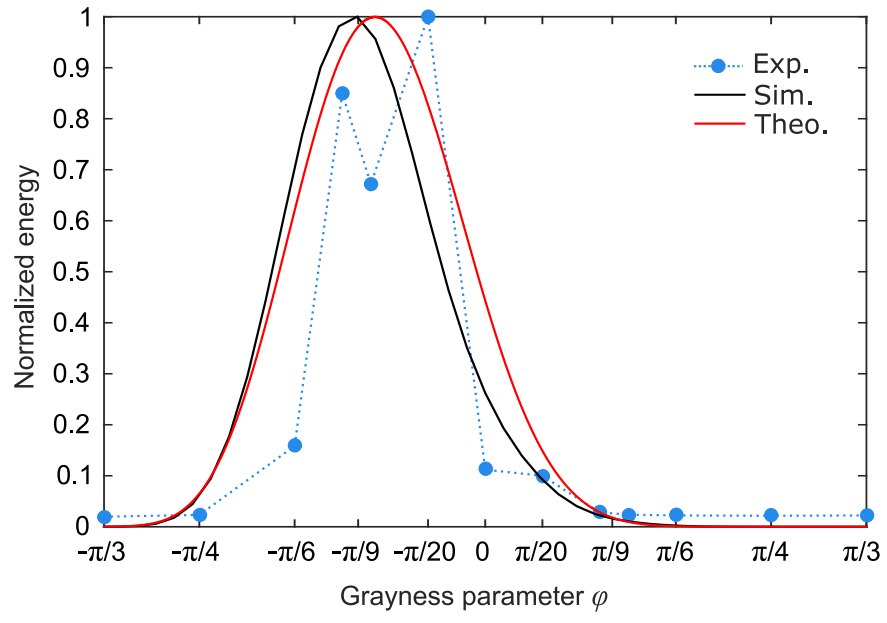

Fig. 4. Evolution of the dispersive wave energy as a function of grayness parameter $\varphi$. The theoretical curve in red line corresponds to the quantity $|G|^{2}\left|\tilde{D}^{\prime}\right|$, the black line corresponds to numerical simulations (see text for details) and full circles represent measurements.

$\tilde{D}^{\prime}(\Omega)=-1 / V_{S}+\beta_{2} \Omega+\frac{\beta_{3}}{2} \Omega^{2}$ is the group velocity of the dispersive wave in the frame co-moving with the soliton, and $\Omega$ is a solution of Eq. (4). Thus Eq. (5) indicates that the dispersive wave amplitude indeed depends on the grayness parameter $\varphi$. Note that a relation similar to Eq. (5) has been found before [see Eq. (4) of Ref. [10]] by developing the approach described in [9].

Figure 4 compares the evolution of radiation amplitude as a function of grayness obtained from theory, numerical simulations [from Fig. 3(b)] and experiments [from Fig. 3(a)]. The experimental and numerical curves were obtained by integrating the spectral power density over the whole dispersive wave spectral peak and dividing by the repetition rate. Thus the plotted quantity is physically equivalent to energy. Since $|G|$ corresponds to the peak amplitude in time domain, we have plotted in Fig. 4 the quantity $|G|^{2}\left|\tilde{D}^{\prime}\right|$, which corresponds to the theoretical energy of the dispersive wave. The three curves were normalized to their maximum. Although the agreement between theory, simulations and experiments is not perfect, the theoretical curve [red line in Fig. 4] still qualitatively shows that the efficiency of dispersive wave generation exhibits an optimum and that it strongly decreases for $\varphi$ values lower than $-\pi / 4$ and greater than $\pi / 9$, as previously observed in experiments and simulations. Indeed, for increasing $\varphi$ values, the phase-matching relation Eq. (4) predicts that the dispersive wave wavelength goes away from the ZDW, as shown by black solid lines in Fig. 3. In this case, the spectral overlap between the soliton spectrum and the anomalous dispersion region decreases, so that the radiation efficiency is reduced and eventually vanishes, similarly to the well-known case of bright solitons [6]. Additionally, for increasing $|\varphi|$ values, the soliton spectrum becomes narrower, which further reduces the spectral overlap across the ZDW so that the radiation efficiency decreases for large negative $\varphi$ values, even if the phase matching curve is closer to the ZDW in this region. Equivalently, the amplitude of the dip in time domain decreases for increasing $|\varphi|$ values, making the source of radiation less efficient, until it becomes totally vanishing (i.e. a pure continuous wave) in the limit $|\varphi|=\pi / 2$.

To summarize, we generated odd-symmetry dark pulses by exploiting commercial waveshapers, and we verified that they can excite dark solitons through cross-correlation and spectral measurements. We studied experimentally, numerically and theoretically the emission of dispersive waves from the synthesized dark solitons as a function of their grayness. We found that the frequency and amplitude of the dispersive waves strongly depend on the soliton grayness, due to the underlying phasematching process.

\section{ACKNOWLEDGEMENT}

This work was partly supported by IRCICA, CNRS, USR 3380, by the NoAWE (ANR-14-ACHN-0014) project, by the "Fonds Européen de Développement Economique Régional", the Labex CEMPI (ANR-11-LABX-0007) and Equipex FLUX (ANR-11EQPX-0017) through the "Programme Investissements d'Avenir". We also acknowledge funding from the CNRS and RFBR (1652-150006) through the Russian-French PRC program. D.V.S. acknowledges support through the ITMO visiting professorship scheme.

\section{REFERENCES}

1. A. Hasegawa and F. Tappert, Appl. Phys. Lett. 23, 142 (1973).

2. A. Hasegawa and F. Tappert, Appl. Phys. Lett. 23, 171 (1973).

3. Y. S. Kivshar and B. Luther-Davies, Physics Reports 298, 81 (1998).

4. P. K. A. Wai, C. R. Menyuk, Y. C. Lee, and H. H. Chen, Opt. Lett., OL 11, 464 (1986).

5. P. K. A. Wai, C. R. Menyuk, H. H. Chen, and Y. C. Lee, Opt. Lett., OL 12, 628 (1987).

6. N. Akhmediev and M. Karlsson, Phys. Rev. A 51, 2602 (1995).

7. I. Cristiani, R. Tediosi, L. Tartara, and V. Degiorgio, Opt. Express, OE 12, 124 (2004).

8. D. V. Skryabin and A. V. Gorbach, Rev. Mod. Phys. 82, 1287 (2010).

9. V. I. Karpman, Physics Letters A 181, 211 (1993).

10. V. V. Afanasjev, Y. S. Kivshar, and C. R. Menyuk, Opt. Lett., OL 21, 1975 (1996).

11. C. Milián, D. V. Skryabin, and A. Ferrando, Opt. Lett., OL 34, 2096 (2009).

12. T. Marest, C. M. Arabí, M. Conforti, A. Mussot, C. Milián, D. V. Skryabin, and A. Kudlinski, Opt. Lett., OL 41, 2454 (2016).

13. P. Emplit, J. P. Hamaide, F. Reynaud, C. Froehly, and A. Barthelemy, Optics Communications 62, 374 (1987).

14. A. M. Weiner, J. P. Heritage, R. J. Hawkins, R. N. Thurston, E. M. Kirschner, D. E. Leaird, and W. J. Tomlinson, Phys. Rev. Lett. 61, 2445 (1988).

15. P. Emplit, M. Haelterman, and J.-P. Hamaide, Opt. Lett., OL 18, 1047 (1993).

16. D. Krökel, N. J. Halas, G. Giuliani, and D. Grischkowsky, Phys. Rev. Lett. 60, 29 (1988).

17. S. A. Gredeskul and Y. S. Kivshar, Phys. Rev. Lett. 62, 977 (1989).

18. S. A. Gredeskul and Y. S. Kivshar, Opt. Lett., OL 14, 1281 (1989).

19. J. P. Heritage, A. M. Weiner, and R. N. Thurston, Opt. Lett., OL 10, 609 (1985).

20. P. Emplit, J.-P. Hamaide, and F. Reynaud, Opt. Lett., OL 17, 1358 (1992).

21. W. J. Tomlinson, R. J. Hawkins, A. M. Weiner, J. P. Heritage, and R. N. Thurston, J. Opt. Soc. Am. B, JOSAB 6, 329 (1989).

22. J. K. Ranka, A. L. Gaeta, A. Baltuska, M. S. Pshenichnikov, and D. A. Wiersma, Opt. Lett. 22, 1344 (1997)

23. M. Conforti and S. Trillo, Opt. Lett. 38, 3815 (2013).

24. M. Conforti, F. Baronio, and S. Trillo, Phys. Rev. A 89, 013807 (2014).

25. F. Biancalana, D. V. Skryabin, and A. V. Yulin, Phys. Rev. E 70, 016615 (2004). 


\section{INFORMATIONAL FIFTH PAGE}

\section{REFERENCES}

1. A. Hasegawa and F. Tappert, "Transmission of stationary nonlinear optical pulses in dispersive dielectric fibers. I. Anomalous dispersion," Appl. Phys. Lett. 23, 142-144 (1973).

2. A. Hasegawa and F. Tappert, "Transmission of stationary nonlinear optical pulses in dispersive dielectric fibers. II. Normal dispersion," Appl. Phys. Lett. 23, 171-172 (1973).

3. Y. S. Kivshar and B. Luther-Davies, "Dark optical solitons: physics and applications," Physics Reports 298, 81-197 (1998).

4. P. K. A. Wai, C. R. Menyuk, Y. C. Lee, and H. H. Chen, "Nonlinear pulse propagation in the neighborhood of the zero-dispersion wavelength of monomode optical fibers," Opt. Lett., OL 11, 464-466 (1986).

5. P. K. A. Wai, C. R. Menyuk, H. H. Chen, and Y. C. Lee, "Soliton at the zero-group-dispersion wavelength of a single-model fiber," Opt. Lett., OL 12, 628-630 (1987).

6. N. Akhmediev and M. Karlsson, "Cherenkov radiation emitted by solitons in optical fibers," Phys. Rev. A 51, 2602-2607 (1995).

7. I. Cristiani, R. Tediosi, L. Tartara, and V. Degiorgio, "Dispersive wave generation by solitons in microstructured optical fibers," Opt. Express, OE 12, 124-135 (2004).

8. D. V. Skryabin and A. V. Gorbach, "Colloquium," Rev. Mod. Phys. 82, 1287-1299 (2010).

9. V. I. Karpman, "Stationary and radiating dark solitons of the third order nonlinear Schrödinger equation," Physics Letters A 181, 211-215 (1993).

10. V. V. Afanasjev, Y. S. Kivshar, and C. R. Menyuk, "Effect of third-order dispersion on dark solitons," Opt. Lett., OL 21, 1975-1977 (1996).

11. C. Milián, D. V. Skryabin, and A. Ferrando, "Continuum generation by dark solitons," Opt. Lett., OL 34, 2096-2098 (2009).

12. T. Marest, C. M. Arabí, M. Conforti, A. Mussot, C. Milián, D. V. Skryabin, and A. Kudlinski, "Emission of dispersive waves from a train of dark solitons in optical fibers," Opt. Lett., OL 41, 2454-2457 (2016).

13. P. Emplit, J. P. Hamaide, F. Reynaud, C. Froehly, and A. Barthelemy, "Picosecond steps and dark pulses through nonlinear single mode fibers," Optics Communications 62, 374-379 (1987).

14. A. M. Weiner, J. P. Heritage, R. J. Hawkins, R. N. Thurston, E. M. Kirschner, D. E. Leaird, and W. J. Tomlinson, "Experimental Observation of the Fundamental Dark Soliton in Optical Fibers," Phys. Rev. Lett. 61, 2445-2448 (1988).

15. P. Emplit, M. Haelterman, and J.-P. Hamaide, "Picosecond dark soliton over a 1-km fiber at 850 nm," Opt. Lett., OL 18, 1047-1049 (1993).

16. D. Krökel, N. J. Halas, G. Giuliani, and D. Grischkowsky, "Dark-Pulse Propagation in Optical Fibers," Phys. Rev. Lett. 60, 29-32 (1988).

17. S. A. Gredeskul and Y. S. Kivshar, "Generation of Dark Solitons in Optical Fibers," Phys. Rev. Lett. 62, 977-977 (1989).

18. S. A. Gredeskul and Y. S. Kivshar, "Dark-soliton generation in optical fibers," Opt. Lett., OL 14, 1281-1283 (1989).

19. J. P. Heritage, A. M. Weiner, and R. N. Thurston, "Picosecond pulse shaping by spectral phase and amplitude manipulation," Opt. Lett., OL 10, 609-611 (1985).

20. P. Emplit, J.-P. Hamaide, and F. Reynaud, "Passive amplitude and phase picosecond pulse shaping," Opt. Lett., OL 17, 1358-1360 (1992).

21. W. J. Tomlinson, R. J. Hawkins, A. M. Weiner, J. P. Heritage, and R. N. Thurston, "Dark optical solitons with finite-width background pulses," J. Opt. Soc. Am. B, JOSAB 6, 329-334 (1989).

22. J. K. Ranka, A. L. Gaeta, A. Baltuska, M. S. Pshenichnikov, and D. A. Wiersma, "Autocorrelation measurement of 6-fs pulses based on the two-photon-induced photocurrent in a GaAsP photodiode," Opt. Lett. 22, 1344 (1997).

23. M. Conforti and S. Trillo, "Dispersive wave emission from wave breaking," Opt. Lett. 38, 3815 (2013).

24. M. Conforti, F. Baronio, and S. Trillo, "Resonant radiation shed by dispersive shock waves," Phys. Rev. A 89, 013807 (2014).

25. F. Biancalana, D. V. Skryabin, and A. V. Yulin, "Theory of the soliton self-frequency shift compensation by the resonant radiation in photonic crystal fibers," Phys. Rev. E 70, 016615 (2004). 